\title{
Development of Chinese chestnut whiskey: yeast strains isolation, fermentation system optimization, and scale-up fermentation
}

\author{
Wanzhen $\mathrm{Li}^{1}$, Cuie Shi ${ }^{2 \dagger}$, Jiaquan Guang ${ }^{3}$, Fei Ge ${ }^{1 *}$ and Shoubao Yan ${ }^{2,3^{*}}$
}

\begin{abstract}
In this study, we used Chinese chestnut as the main raw material to develop a novel type of whiskey. First, 16 yeasts were isolated and identified for producing aroma using olfactory plate assay. Of these, we screened nine yeast strains based on their fermentation capacity, aroma profile, and sensory evaluation. The results demonstrated the combination of strains HN006 (Saccharomyces cerevisiae) and HN010 (Wickerhamomyces anomalus) provided satisfactory wine fermentation with an interesting flavor profile, as strain HN010 was highly aromatic and had elevated sensory scores with comparatively low ethanol yield, while strain HN006 had a poor flavor profile but produced the largest amount of ethanol. Subsequently, we co-cultured strains HN006 and HNO10 to optimize the fermentation system. The results revealed the following optimum parameters: a mixed inoculum of $6 \%(\mathrm{~V} / \mathrm{v})$ at an HN006/HN010 ratio of 1:2 (v/v), a raw material ratio of 5:3:2 (chestnut: malt: glutinous rice), and yeast extract concentration of $6 \mathrm{~g} / \mathrm{L}$. Additionally, this fermentation system was successfully scaled-up to a $1000 \mathrm{~L}$ pilot-scale system. The results of this study showed that strains HN006 and HN010 could be used as alternatives for whiskey fermentation, as well as provided a generalized experimental scheme to assess other microorganisms.
\end{abstract}

Keywords: Chinese chestnut, Whisky, Volatile flavor compounds, Fermentation

\section{Introduction}

Chinese chestnut, one of the major nuts in China, belongs to the genus Castanea Miller and the family Fagaceae and is also known as "the king of dry fruit" (Zhang et al. 2018a, b). Its edible kernels are popular in China and are rich in both macronutrients (starch, protein, and fat) and micronutrients (minerals, such as iron, calcium, phosphorus, and several types of vitamins, as well as trace minerals). Modern pharmacological studies have shown that the Chinese chestnut nourishes the stomach and the spleen, improves the functioning of kidneys, strengthens the tendons, activates blood circulation, and is used for

\footnotetext{
*Correspondence: 1176144756@qq.com

${ }^{\dagger}$ Cuie Shi Co-first author

${ }^{1}$ College of Biological and Food Engineering, Anhui Polytechnic

University, Anhui 241000, People's Republic of China

${ }^{2}$ School of Life Science, Huainan Normal University, Huainan, Anhui

232001, People's Republic of China

Full list of author information is available at the end of the article
}

the prevention and treatment of hypertension and coronary heart disease (Zhang et al. 2014).

Chinese chestnut is a cultivated plant with a history dating back approximately 3000 years in China, and ever since, there has been an annual increase in the production of chestnuts (Zhang et al. 2018a, b). However, the phenomenon of "difficult to sell chestnut" still exists in many planting areas in China, which has reduced the motivation of the chestnut farmers. Additionally, the seasonal availability of Chinese chestnut fruits along with their high water content (approximately $50 \%$ on a wet basis) limits their storage due to germination, mildew, and insect infestation (Sheng and Yu 2015). Currently, the main processing methods of Chinese chestnuts include baking, cooking, or frying with sugar (tang chao li zi), which is not beneficial economically. Thus, there is an urgent need to identify and develop novel processing methods for using Chinese chestnuts to produce highvalue products, such as Chinese chestnut wine.
Springer Open
C The Author(s) 2021. This article is licensed under a Creative Commons Attribution 4.0 International License, which permits use, sharing, adaptation, distribution and reproduction in any medium or format, as long as you give appropriate credit to the original author(s) and the source, provide a link to the Creative Commons licence, and indicate if changes were made. The images or other third party material in this article are included in the article's Creative Commons licence, unless indicated otherwise in a credit line to the material. If material is not included in the article's Creative Commons licence and your intended use is not permitted by statutory regulation or exceeds the permitted use, you will need to obtain permission directly from the copyright holder. To view a copy of this licence, visit http://creativeco mmons.org/licenses/by/4.0/. 
Whiskey is one of the four famous distilled wines in the world and is popular internationally. The most famous whiskey producing countries are Scotland, Ireland, the USA, Japan, and Canada. However, there are few reports on whiskey production in China. The high starch content of the Chinese chestnut makes it suitable for whiskey brewing. However, Chinese chestnut whiskey (wine) is still scarce in the domestic and foreign markets.

The sensory attributes of whiskey result from compounds that originate from the raw materials, the metabolites of yeast, and maturation process. Among them, the yeast make a special contribution to the flavour quality of whiskey, and the selection of microorganisms is critical for the formation of aroma, which is one of the key attributes affecting consumer acceptance (Du et al. 2019).

Therefore, this study focused on developing a novel whiskey using the Chinese chestnut as the main raw material. We isolated and investigated the potential of specific yeast strains for effective Chinese chestnut wine fermentation. Additionally, we used the screened strains to optimize the fermentation process based on high ethanol yield and sensory scores. Furthermore, we used a $1000 \mathrm{~L}$ pilot-scale system to scale-up the fermentation to industrialize the process of Chinese chestnut wine production. The results of this study would provide supportive evidence to develop a Chinese chestnut-based novel whiskey.

\section{Materials and methods Materials}

Chinese chestnuts and glutinous rice were purchased from a retail market in the Huoshan county, Anhui province of China, in October 2019. The malt was obtained from Zhengzhou Longhai beer Materials Co., Ltd (Zhengzhou, Henan province, China). The thermostable $\alpha$-amylase and glucoamylase for saccharification were acquired from the Sunson Industry Group Co., Ltd. (China).

\section{Isolation of superior yeast strain Initial screening for yeast strains}

In a $250 \mathrm{~mL}$ flask, we mixed $5 \mathrm{~g}$ of Chinese strong flavour Daqu, which is a saccharifying and fermenting agent utilized in the brewing of Chinese strong flavour liquor and contains a variety of yeast species, into $100 \mathrm{~mL}$ of yeast extract peptone dextrose medium (YEPD; $5 \mathrm{~g} / \mathrm{L}$ yeast extract $+50 \mathrm{~g} / \mathrm{L}$ glucose $+10 \mathrm{~g} / \mathrm{L}$ peptone). The mixture was stirred at $200 \mathrm{rpm}$ at $30{ }^{\circ} \mathrm{C}$ for $24 \mathrm{~h}$. The culture was serially diluted with sterile water and coated on the YEPD plates supplemented with $0.01 \%$ chloromycetin in duplicates. Next, 48-72 h later, we isolated colonies with varying morphologies and purified them, followed by inoculation on a YEPD agar plate to conduct a smelling or "sniffing" test for initial aroma screening following the method of Alicia et al. (2018).

\section{Repetitive element sequence-based PCR (Rep-PCR) analysis and yeast identification}

A DNA extraction kit was used to extract the genomic DNA of the isolated yeast strains (Sangon Biotech, Shanghai, China). The rep-PCR analysis was done using the primer $(\text { GTG })_{5}$ (5'-GTGGTGGTGGTGGTG-3') using a previously described method (Simonetta et al. 2016). A 1.2\% agarose gel electrophoresis (AGE) was used to separate the amplicons in a $1 \times$ TBE buffer $(150 \mathrm{~min}$, $120 \mathrm{~V})$. The clusters were identified by analyzing the resultant dendrograms.

For identification of the yeast, the universal primer pairs NL1 and NL4 were used to sequence the strain of each rep-PCR fingerprint group (Yan et al. 2019a, b), followed by alignment to the $26 \mathrm{~S}$ rRNA gene sequences in the GenBank database using the BLAST algorithm tool. The nucleotide sequences obtained in this study have been assigned GenBank Accession Nos. MW076944MW076959, as indicated in Fig. 1.

\section{Testing for the formation of volatile flavor compounds}

Chinese chestnuts were peeled, cleaned, and steamcooked for $30 \mathrm{~min}$. After naturally cooling to room temperature, the cooked chestnut kernels were mixed with water $(1: 3(\mathrm{~m} / \mathrm{v}))$ and pulped using a fruit mixer. Next, the mixtures were transferred into a bioreactor and saccharified using $\alpha$-amylase $(10 \mathrm{U} / \mathrm{g}$ chestnut kernel; $\mathrm{pH}$ 5.5, $90{ }^{\circ} \mathrm{C}, 30 \mathrm{~min}$ ) and subsequently, glucoamylase (200 U/g chestnut kernel; pH 4.5, $60{ }^{\circ} \mathrm{C}, 6 \mathrm{~h}$ ). Then, a double gauze was used to separate the liquid phase of the saccharified mixture and placed into a $2.5 \mathrm{~L}$ glass bottle, each with $2 \mathrm{~L}$ volume. The liquid phase of the saccharified mixture was sterilized at $113^{\circ} \mathrm{C}$ for $10 \mathrm{~min}$ and subjected to ethanol fermentation. The conditions of the fermentation and distillation of Chinese chestnut wine have been listed in Sect. 2.3. Finally, the volatile compounds in each sample of the obtained raw wine were detected and quantified by solid-phase microextraction-gas chromatography/mass spectrometry (SPME-GC-MS).

\section{Testing for fermentation ability of isolated strains}

The seed colonies of each isolated yeast strain were cultured aerobically in liquid YEPD medium for $24 \mathrm{~h}$ at $30{ }^{\circ} \mathrm{C}$ with constant stirring at $200 \mathrm{rpm}$. Next, 5\% (v/v) of these suspension cultures (approximately $1 \times 10^{7} \mathrm{CFU} /$ $\mathrm{mL}$ ) were added to $250 \mathrm{~mL}$ Erlenmeyer flasks containing YEPD medium $(200 \mathrm{~mL})$ with $190 \mathrm{~g} / \mathrm{L}$ glucose. The flasks had perforated silicon stoppers equipped with $0.45 \mathrm{~mm}$ filters (Merck Millipore, Italy), to release carbon dioxide and to prevent contamination. All the tests were carried 


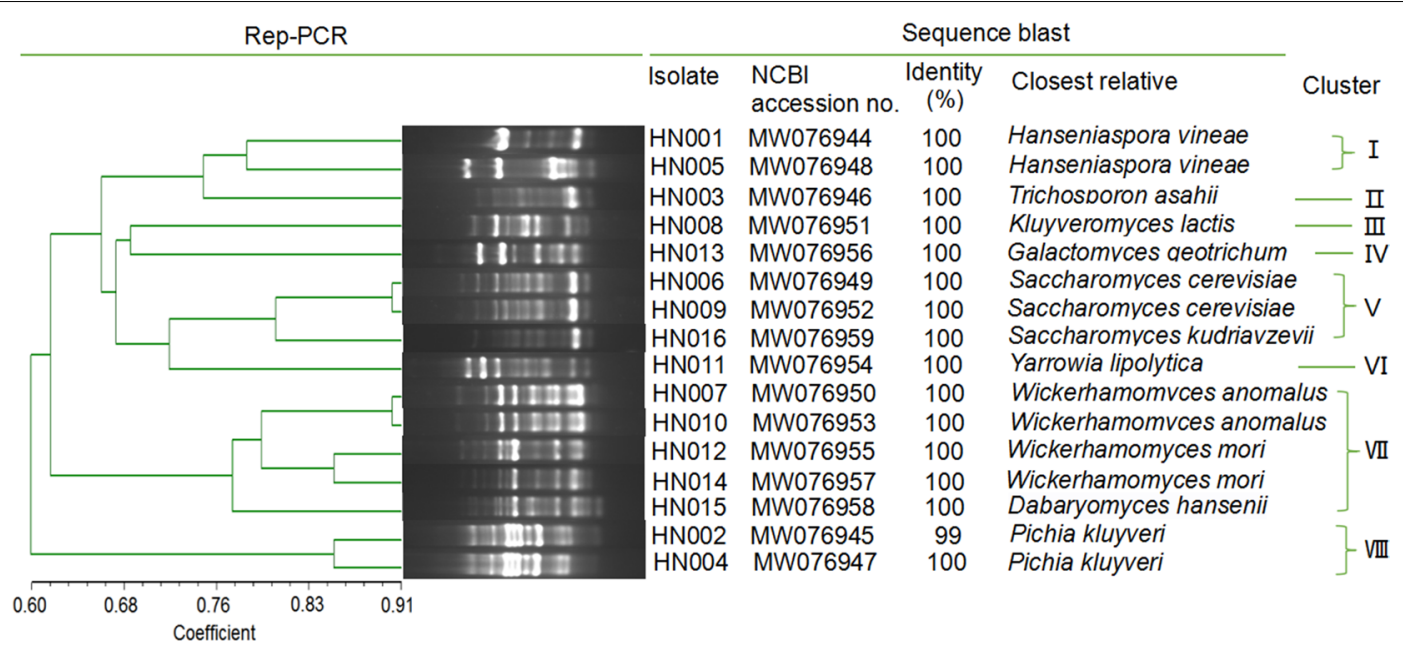

Fig. 1 Dendrogram obtained by cluster analysis of (GTG) $)_{5}$-based rep-PCR fingerprints of the isolated yeasts, based on Dice's coefficient of similarity with the unweighted pair group method with arithmetic average clustering algorithm (UPGMA). Yeast species were identified by sequencing of 265 rRNA gene and GenBank searches

out in triplicates and performed under static conditions at $30^{\circ} \mathrm{C}$. Post-fermentation, each fermentation broth was sampled to detect the remaining reducing sugar content, as well as the ethanol content.

\section{Sensory evaluation of the flavor of yeast fermentation products}

Section 2.6 describes the methods used for the evaluation of the sensory features, including smell, taste, flavor, acidity, and overall acceptability of each obtained distillate. Further testing was done using yeast strains with high total scores.

\section{Laboratory fermentation of Chinese chestnut wine}

Additional file 1: Figure S1 shows the manufacturing process of Chinese chestnut whiskey raw wine. The Chinese chestnut kernel, malt, and glutinous rice were cleaned and soaked (glutinous rice was soaked for $2 \mathrm{~h}$ in 1.5 times water). Post-soaking, glutinous rice and Chinese chestnut kernels were steam-cooked for $30 \mathrm{~min}$ and cooled to room temperature. Then, water was added at a ratio of $1: 3(\mathrm{~m} / \mathrm{v})$ and the mixture was saccharified by $\alpha$-amylase $(10 \mathrm{U} / \mathrm{g}$ raw material $)$ at $\mathrm{pH}$ $5.5,90{ }^{\circ} \mathrm{C}$ for $30 \mathrm{~min}$; followed by saccharification with glucoamylase at $\mathrm{pH} 4.5,60{ }^{\circ} \mathrm{C}$ for $6 \mathrm{~h}$ with the addition of the pre-crushed malt. Subsequently, a double gauze was used to separate the liquid phase of the saccharified mixture, followed by adjusting the $\mathrm{pH}$ to 5.0 and transferring the contents into a $5 \mathrm{~L}$ fermenter with $4 \mathrm{~L}$ of working volume. After sterilization at $113{ }^{\circ} \mathrm{C}$ for $10 \mathrm{~min}$, the prepared sugar solution was subjected to wine fermentation by inoculation with pre-cultured yeast seeds. The temperature of the fermentation system was maintained at $30^{\circ} \mathrm{C}$.

Post-alcoholic fermentation, we immediately doubledistilled the fermented liquids in a $5 \mathrm{~L}$ Charentais potstill. When the alcohol concentration was less than $0.5 \%(\mathrm{w} / \mathrm{v})$, we stopped the first distillation, resulting in an approximately 20\% (v/v) distillate. During the second distillation, approximately one-third of the distillate was collected, resulting in a final alcohol concentration of $60 \%(\mathrm{v} / \mathrm{v})$ in the final raw Chinese chestnut wine.

\section{Optimization of Chinese chestnuts wine fermentation}

We performed anaerobic Chinese chestnut wine fermentation as described previously for 6 days to optimize the fermentation process. At the end of the process, we collected samples of fermentation broth to determine the ethanol and residual sugar content, as well as to evaluate the overall sensory features of the obtained wine. We also evaluated the impact of different inoculum sizes on the production of Chinese chestnuts wine. Table 2 shows the settings used for this analysis. Additionally, we examined the impact of different raw materials on the brewing of chestnut whiskey. The Chinese chestnut kernels, malt, and glutinous rice were prepared in various ratios (Table 3) and were subjected to the wine fermentation process following the previously described procedure. Furthermore, we also tested different supplementation amounts of nitrogen nutrient (yeast extract). All experiments were conducted in triplicates, and the results were expressed as the average of the three repetitions. 


\section{The inoculum preparation for $1000 \mathrm{~L}$ pilot-scale fermentation}

For inoculum preparation, the suspended cells of the obtained yeast from single colonies on YEPD agar plates were pre-cultured in $250 \mathrm{~mL}$ flasks containing $100 \mathrm{~mL}$ YEPD medium at $30{ }^{\circ} \mathrm{C}$ for $24 \mathrm{~h}$. Then each of $20 \mathrm{~mL}$ cultures were transferred to five $500 \mathrm{~mL}$ flasks each containing $180 \mathrm{~mL}$ YEPD medium, and cultivated under the same conditions mentioned above, after which the entire volume of the five $500 \mathrm{~mL}$-flasks was used to inoculate a $10 \mathrm{~L}$ fermentor $(\mathrm{v} / \mathrm{v}, 10 \%)$. The culture broth from the $10 \mathrm{~L}$ fermentor was transferred to a $150 \mathrm{~L}$ fermentor containing $100 \mathrm{~L}$ of growth medium. Both the fermentation scales of 10 and $150 \mathrm{~L}$ were conducted at $30{ }^{\circ} \mathrm{C}, 300 \mathrm{rpm}$, and VVM of 4.0 for $24 \mathrm{~h}$. Finally the culture broth from $150 \mathrm{~L}$ fermentor was used for the pilot-scale fermentation. The volumes to be inoculated were determined on the basis of cell counting under a light microscope with a Thoma Zeiss chamber.

\section{$1000 \mathrm{~L}$ pilot-scale fermentation}

We performed the pilot scaling-up experiments based on the optimization conditions derived from laboratory experiments in a pilot-scale plant built at Anhui Yingjia Distillery Co., Ltd., located in Huoshan county, Luan City, China. The scale-up fermentation was performed in a $1000 \mathrm{~L}$ fermenter with a liquid loading capacity of $900 \mathrm{~L}$. Additional file 1: Figure S2 shows a schematic diagram of the experimental apparatus used in this study.

\section{Analytical methods}

During the fermentation process, we collected broth samples at specific time intervals, followed by 20 -min centrifugation at $5000 \mathrm{~g}$ to remove the cellular debris. The 3, 5-dinitro-salicylic acid colorimetric method was used to determine the reducing sugar content (Miller 1959). The measurement of yeast cells and the ethanol content was performed following the method of Yan et al. (2013).

\section{Analyses of the volatile compounds}

The HS-SPME-GC-MS was done to analyze the aroma profiles of the obtained wine samples, following a previously described method (Yan et al. 2019a, b). The volatile flavor compounds in the samples were identified by comparing the mass spectrum data with the NIST 05a MS database (Agilent Technologies, Inc., USA), and quantification was done based on the comparison of their peak intensity to that of the 2-octanol (internal reference).

Additionally, heatmap clustering was used to analyze the HS-SPME-GC-MS dataset to identify the differences in the fermentation profiles of different yeast strains. The analysis was done using $\mathrm{R}$ (programming language) Software (Annalisa et al. 2018).

\section{Sensory evaluation}

We evaluated the sensory characteristics of smell, taste, acidity, after-taste, and overall acceptability of the final Chinese chestnuts wine following a previously reported method (Yan et al. 2019a, b). Wine samples (20 mL) were labeled with a randomly assigned 3-digit code and simultaneously served in ISO tasting glasses covered with plastic Petri dishes at room temperature.

\section{Results}

Preliminary screening of yeast strains

Post-morphological examination (both micro and macro), we identified 16 isolates that were growing on YEPD agar as yeast strains. The cluster analysis of the rep-PCR of these 16, based on a coefficient of similarity of $76 \%$, resulted in eight clusters (Fig. 1). We identified the isolates in each cluster by sequencing the D1/ D2 region of the $26 \mathrm{~S}$ rRNA gene, followed by a BLAST search at GenBank (Fig. 1). Approximately $31.25 \%$ of the yeast isolates were found in cluster VII, which were identified as Wickerhamomyces anomalus (HN007, HN010), Wickerhamomyces mori (HN012, HN015), and Dabaryomyces hansenii (HN014). Approximately 18.75\% of the yeast isolates were found in cluster $\mathrm{V}$ and shared $100 \%$ homology with GenBank sequences of Saccharomyces cerevisiae (HN006, HN009) and Saccharomyces kudriavzevii (HN016). The cluster I and VIII isolates were unambiguously identified as Hanseniaspora vineae (HN001, HN005) and Pichia kluyveri (HN002, HN004), respectively. The remaining four clusters (II, III, IV, and VI) were identified as Trichosporon asahii (HN003), Kluyveromyces lactis (HN008), Galactomyces geotrichum (HN013), and Yarrowia lipolytica (HN011), respectively, each showing $100 \%$ homology to GenBank sequences.

We performed the olfactory "sniff" test on the YEPD medium plates to preliminarily determine the aroma producing ability of the obtained 16 yeast strains. Table 1 presents the results of this analysis. We found that different genera of the isolates presented various aromas, differentiated by different intensities of sourness, fruity, floral, cheese-like, and cream-like smells. Saccharomyces cerevisiae (HN006, HN009) and Saccharomyces kudriavzevii (HN016) produced different intensities of alcoholic aroma in the YEPD agar plates. Both Dabaryomyces hansenii (HN014), and Yarrowia lipolytica (HN011) produced characteristic cheese-like smells with intermediate strength. Each of the following strains: Trichosporon asahii (HN003), Wickerhamomyces anomalus (HN007, HN010), Wickerhamomyces mori (HN012, HN015), Pichia kluyveri (HN002, HN004), and Kluyveromyces lactis (HN008) produced pleasant fragrance of pineapple fruit, apple and rose fruit, apple fruit, banana fruit, and strawberry fruit, respectively, and among which 
Table 1 The pleasant aroma characteristics of the obtained strains by agar plate "sniff" assay method

\begin{tabular}{|c|c|c|c|}
\hline Strain code & Closest relative $^{1}$ & Aroma & Aroma intensity $^{2}$ \\
\hline HN001 & Hanseniaspora vineae & Sour aroma & + \\
\hline HN002 & Pichia kluyveri & Banana fruity aroma & ++ \\
\hline HN003 & Trichosporon asahii & Pineapple fruity aroma & ++ \\
\hline HN004 & Pichia kluyveri & Banana fruity aroma & + \\
\hline HN005 & Hanseniaspora vineae & Sour aroma & + \\
\hline HN006 & Saccharomyces cerevisiae & Alcohol aroma & ++ \\
\hline HN007 & Wickerhamomyces anomalus & Apple fruity aroma & ++ \\
\hline HN008 & Kluyveromyces lactis & Strawberry fruity aroma & ++ \\
\hline HN009 & Saccharomyces cerevisiae & Alcohol aroma & + \\
\hline HN010 & Wickerhamomyces anomalus & Apple fruity and rose aroma & +++ \\
\hline HN011 & Yarrowia lipolytica & Cheese and sweet aroma & ++ \\
\hline HN012 & Wickerhamomyces mori & Apple fruity aroma & + \\
\hline HN013 & Galactomyces geotrichum & Cream aroma & ++ \\
\hline HN014 & Dabaryomyces hansenii & Cheese aroma & ++ \\
\hline HN015 & Wickerhamomyces mori & Apple fruity aroma & + \\
\hline HN016 & Saccharomyces kudriavzevii & Alcohol aroma and slight sour aroma & + \\
\hline
\end{tabular}

1 The Closest relative was in corresponding with that of Fig. 1

2 Weak $(+)$, intermediate $(++)$, strong $(+++)$

strain Wickerhamomyces anomalus (HN010) possessed the highest intensity. While the strain Hanseniaspora vineae (HN001, HN005) generated an unpleasant sour flavor, the strain Galactomyces geotrichum (HN013) produced a cream-like aroma, which was reported to have wide application in the fermentation of dairy products (Jacques et al. 2017).

Based on these results, nine strains (HNO02, HNO03, HN006, HN008, HN009, HN010, HN011, HN013, and HN014), which produced a pleasant aroma with moderate and strong intensity were selected for the follow-up experimental studies.

These nine strains were further examined for Chinese chestnuts wine fermentation, and each aroma profile of the obtained wine was measured by the SPME-GC-MS technique to further evaluate their performance in the accumulation of aroma compounds for the production of the Chinese chestnuts wine. Table 2 presents the list of all identified volatile compounds, which were mainly characterized by the following functional groups: 10 volatile acids $(6.744-14.721 \mu \mathrm{g} / \mathrm{mL}), 14$ esters $(3.502-16.456 \mu \mathrm{g} /$ $\mathrm{mL}), 11$ alcohols $(1.890-8.689 \mu \mathrm{g} / \mathrm{mL}), 6$ aldehydes $(0.309-3.974 \mu \mathrm{g} / \mathrm{mL}), 3$ ketones $(0.108-0.604 \mu \mathrm{g} / \mathrm{mL}), 3$ Alkanes $(0.373-1.188 \mu \mathrm{g} / \mathrm{mL})$, and, at a lower content, by 2 volatile phenols $(0-0.409 \mu \mathrm{g} / \mathrm{mL})$.

Apart from presenting the overall aromatic profile of each tested strain, Table 2 also highlighted the differential behavior exhibited by strains belonging to the same species. We observed a variation in the total concentration of each type of volatile compounds based on different strains. The samples produced by non-Saccharomyces yeasts (HN002, HN003, HN008, HN010, HN011, HN013, and HN014) possessed higher amounts of volatile acids compared with those of Saccharomyces yeasts (HN006, HN009), especially the wine fermentation using the strain HN010 showed the highest concentration $(15.721 \mu \mathrm{g} / \mathrm{mL})$ of various acids, with the exception of butanoic acid, hexanoic acid, 2-methyl-butanoic acid, and nonanoic acid. Butanoic acid and hexanoic acid are generally present in the Chinese strong flavor liquor, and their high content can impart an unpleasant flavor to the wine (Aslankoohi et al. 2016). The presence of 2-methylbutanoic acid in the strains HNO11 and HNO14 imparts cheese-like and sweet characteristics (Marycarmen et al. 2020), which were consistent with the results of our preliminary olfactory "sniff" test. Nonanoic acid, detected only in strain HN009, had green and fat characteristics (Aslankoohi et al. 2016). No significant difference was observed in the type and concentration of volatile acids between HN006 and HN009.

We observed a significant difference in the concentrations of esters in the wines produced using different cultures (Table 2). We found a significantly higher concentration of esters in the non-Saccharomyces culture wines compared with the Saccharomyces culture wines, which was probably attributed to its low lipase activity (Table 2). The highest concentration of esters $(16.456 \mu \mathrm{g} / \mathrm{mL})$ was found in the wine prepared using HN010, which was 3.22 and 3.70 times higher than that in the Saccharomyces yeast strain of HN006, HN009, 


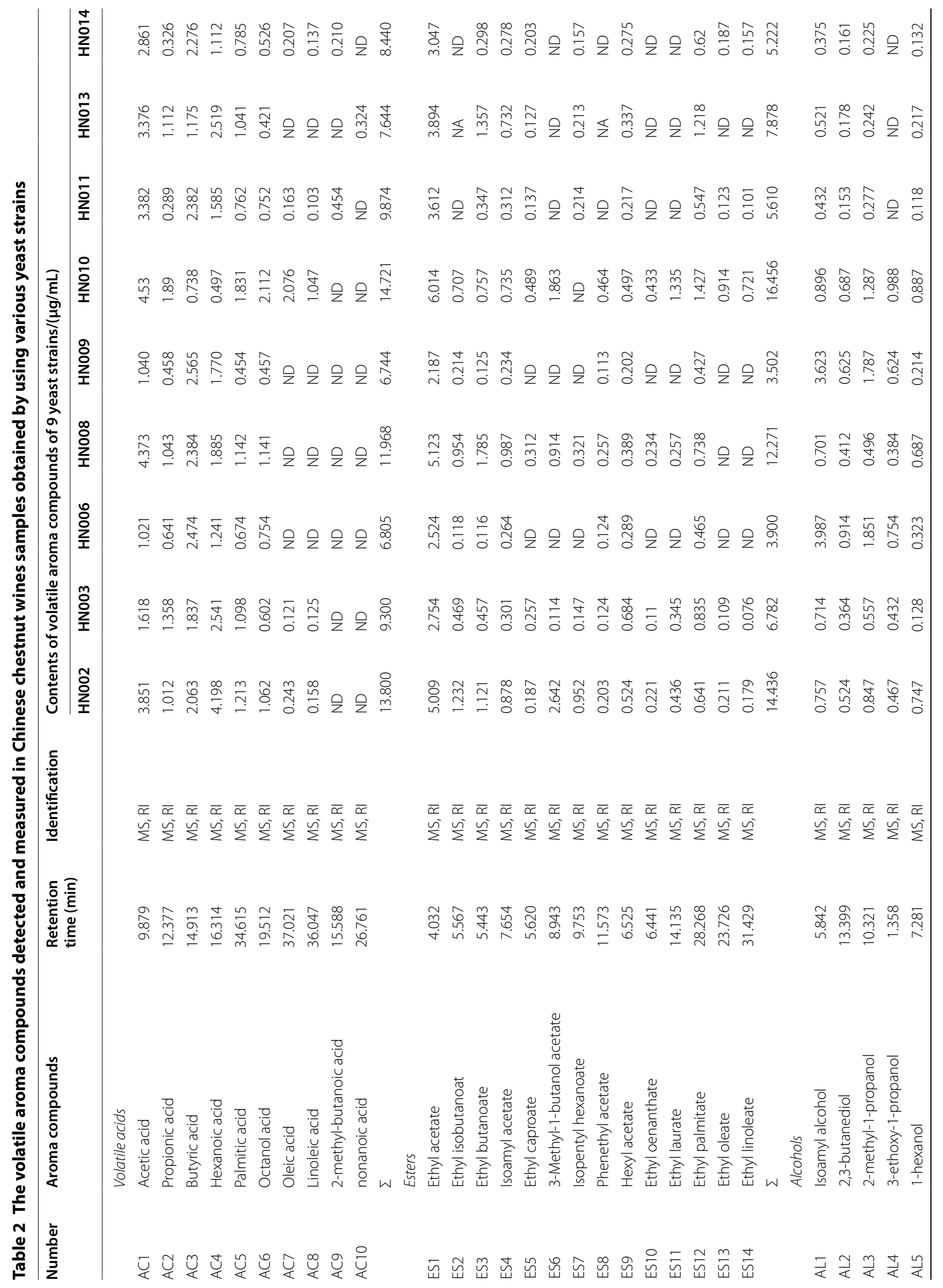




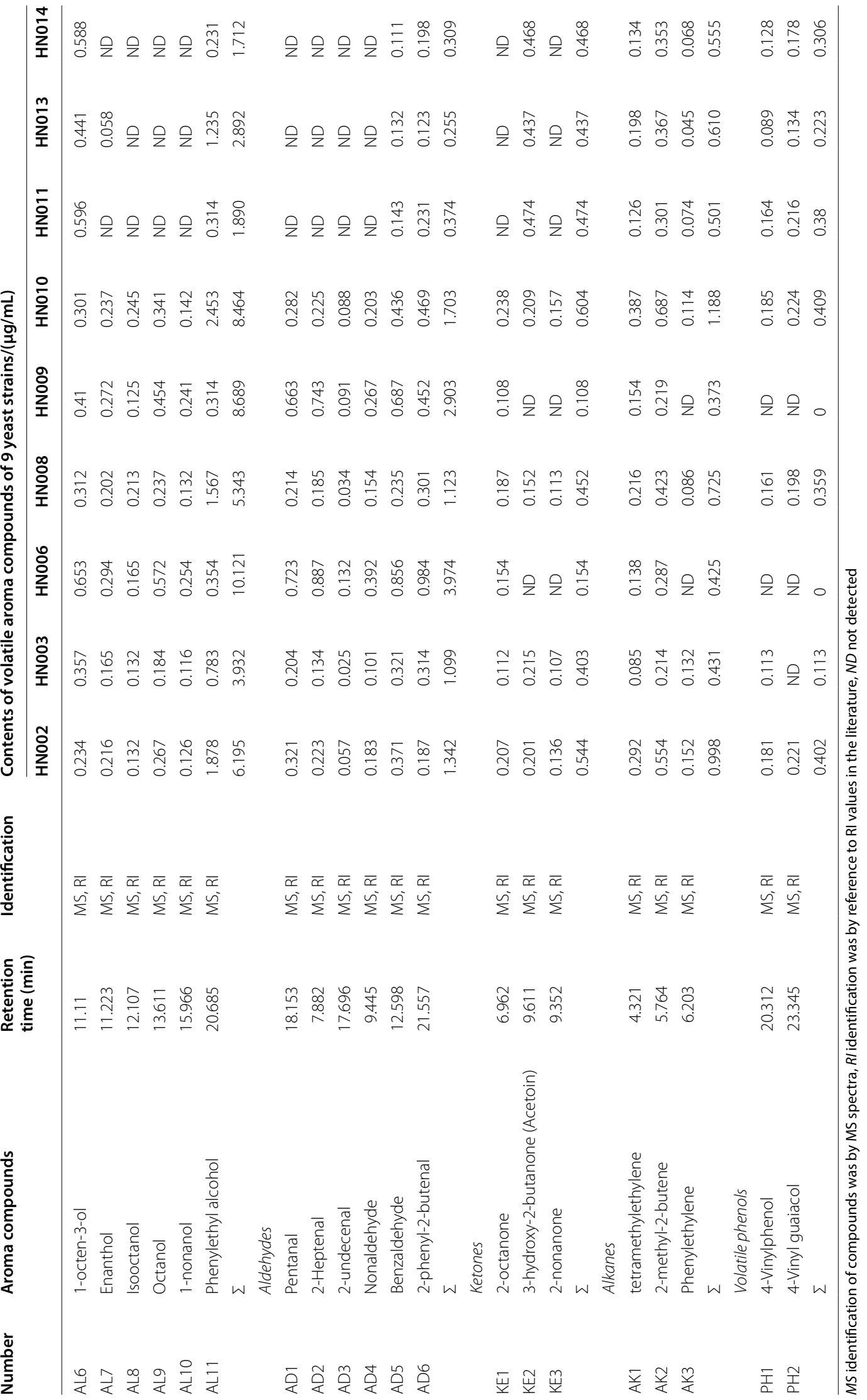


respectively. Amongst all types of esters, the concentration of ethyl acetate was found to be the highest for each strain, ranging from $2.187 \mu \mathrm{g} / \mathrm{mL}$ (HN006) to $16.456 \mu \mathrm{g} / \mathrm{mL}$ (HN010). Ethyl esters, typically described to possess a "fruity and flower" aroma (Laura et al. 2019), were the largest ester-based compounds in the Chinese chestnut wine. We found extremely high amounts of ethyl acetate (sweet, fruity), phenylethyl acetate (rosy), and ethyl caproate (apple peel, fruity) in the Chinese chestnut wine produced by HNO10 (Marycarmen et al. 2020), along with high-molecular-weight ethyl esters, such as ethyl laurate, ethyl palmitate, ethyl oleate, and ethyl linoleate, which were reported to promote the accumulation of the after-taste in the Chinese strong flavor liquor (Yan et al. 2019a, b).

Higher alcohols, produced by yeast, originate either from the degradation of branched-chain amino acids or directly from sugar fermentation. We found that the higher alcohols constituted the major quantitative component in Saccharomyces (HN006, HN009)-fermented chestnut wine samples, and were significantly higher than those obtained by non-Saccharomyces-fermented wines (Table 2). The wine samples brewed using the strain HN006 exhibited the highest concentration of total higher alcohols, which included isoamyl alcohol $(3.987 \mu \mathrm{g} / \mathrm{mL}), 1$-nonanol $(0.254 \mu \mathrm{g} / \mathrm{mL})$, 1-hexanol $(0.323 \mu \mathrm{g} / \mathrm{mL}), 2,3$-butanediol $(0.914 \mu \mathrm{g} / \mathrm{mL})$, isooctanol $(0.165 \mu \mathrm{g} / \mathrm{mL}), 2$-methyl-1-propanol $(1.851 \mu \mathrm{g} /$ $\mathrm{mL})$, enanthol $(0.294 \mu \mathrm{g} / \mathrm{mL})$, 3-ethoxy-1-propanol $(0.754 \mu \mathrm{g} / \mathrm{mL})$, 1-octen-3-ol $(0.653 \mu \mathrm{g} / \mathrm{mL})$, octanol $(0.572 \mu \mathrm{g} / \mathrm{mL})$, and phenylethyl alcohol $(0.354 \mu \mathrm{g} / \mathrm{mL})$. Of these, isoamyl alcohol, 2,3-butanediol, and phenylethyl alcohol have previously been reported to be present in fermented alcoholic beverages as characteristic flavor compounds, such as Chinese strong flavor liquor (Yan et al. 2019a, b).

Aldehydes are produced from amino acids either by Strecker degradation or by transamination, followed by decarboxylation, and they can be easily reduced to alcohols. We observed that the Saccharomyces-fermented wines possessed a significantly higher concentration of aldehydes compared with the non-Saccharomyces-fermented wines. This trend was consistent with the trends of higher alcohols (Table 2).

Additionally, we detected three aldehydes in the Chinese chestnut wine samples, and they were found to be most abundant in the strain HN006. Among these, 2-octanone, which imparts a cream-like flavor to the wine body (Yan and Dong 2019), was found in the Chinese chestnut wine samples. Also, 2-nonanone, which has a fruity and flowery note (Yan and Dong 2019), was found in each chestnut wine sample fermented using strains HN005, HN006, HN007, and HN008.
We found the highest levels of alkanes $(1.188 \mu \mathrm{g} / \mathrm{mL})$ in the samples fermented by strain HN006, and they constituted tetramethylethylene $(0.387 \mu \mathrm{g} / \mathrm{mL}), 2$-methyl2-butene $(0.687 \mu \mathrm{g} / \mathrm{mL})$, and phenylethylene $(0.114 \mu \mathrm{g} /$ $\mathrm{mL}$ ). Additionally, two volatile phenols, namely 4-vinylphenol, and 4-vinylguaiacol, were also identified to be present in the highest amount in the samples with strain HN006, and are known to possess a characteristic spicelike aroma (Yan et al. 2019a, b).

We created a heatmap representation to allow a rapid visual assessment of the similarities and differences in the volatile profile of the strains between different samples. Additionally, the acids, alcohols, and esters represented the major functional groups for all the yeast strains (Fig. 2). Figure 2 shows the differences in the relative abundance of compounds among different strains, revealing the presence of different flavor profiles in different strains. For instance, the Saccharomyces strains (HN006, HN009), produced high levels of ethyl esters, isoamyl alcohol, and 2-methyl-1-propanol, along with the non-Saccharomyces strains (HN004 and HN010). Notably, the strain HNO10 showed relatively larger amounts of volatile compounds compared with other strains, which indicated that it might produce wine with a more harmonious wine aroma. These results also suggested that different yeast strains, especially non-Saccharomyces yeasts, imparted a specific influence on wine flavor. However, their fermentation characteristics are not yet fully understood, and further research is required to identify the wine fermentation capacity of each strain.

\section{Testing for fermentation ability}

We tested the fermentation performance of the obtained nine yeast strains to identify the optimal yeast strain for preparing the Chinese chestnut wine. Figure 3a, b show the effects of yeast strains on the concentrations of ethanol and reducing sugar during the fermentation of Chinese chestnut wine. We observed a slight increase in the concentration of ethanol for Saccharomyces strains (HN006, HN009) during the initial $24 \mathrm{~h}$ of fermentation, which increased significantly and reached the maximum value after $120 \mathrm{~h}$, whereas the ethanol content produced by non-Saccharomyces strains (HN002, HN003, HN008, HN010, HN011, HN013, and HN014) got slowly accumulated throughout the fermentation, and their corresponding concentrations were lower than those produced by the Saccharomyces strains. Table 3 also summarizes the kinetic parameters of the ethanol fermentation with different strains. The strain HN006 generated the maximum concentration of ethanol $(90.220 \pm 0.874 \mathrm{~g} / \mathrm{L})$, had the highest ethanol productivity $(0.752 \pm 0.026 \mathrm{~g} / \mathrm{L} / \mathrm{h})$, and sugar utilization (99.5\%) after $120 \mathrm{~h}$ (Table 3). Also, this strain achieved the highest final ethanol yield of 


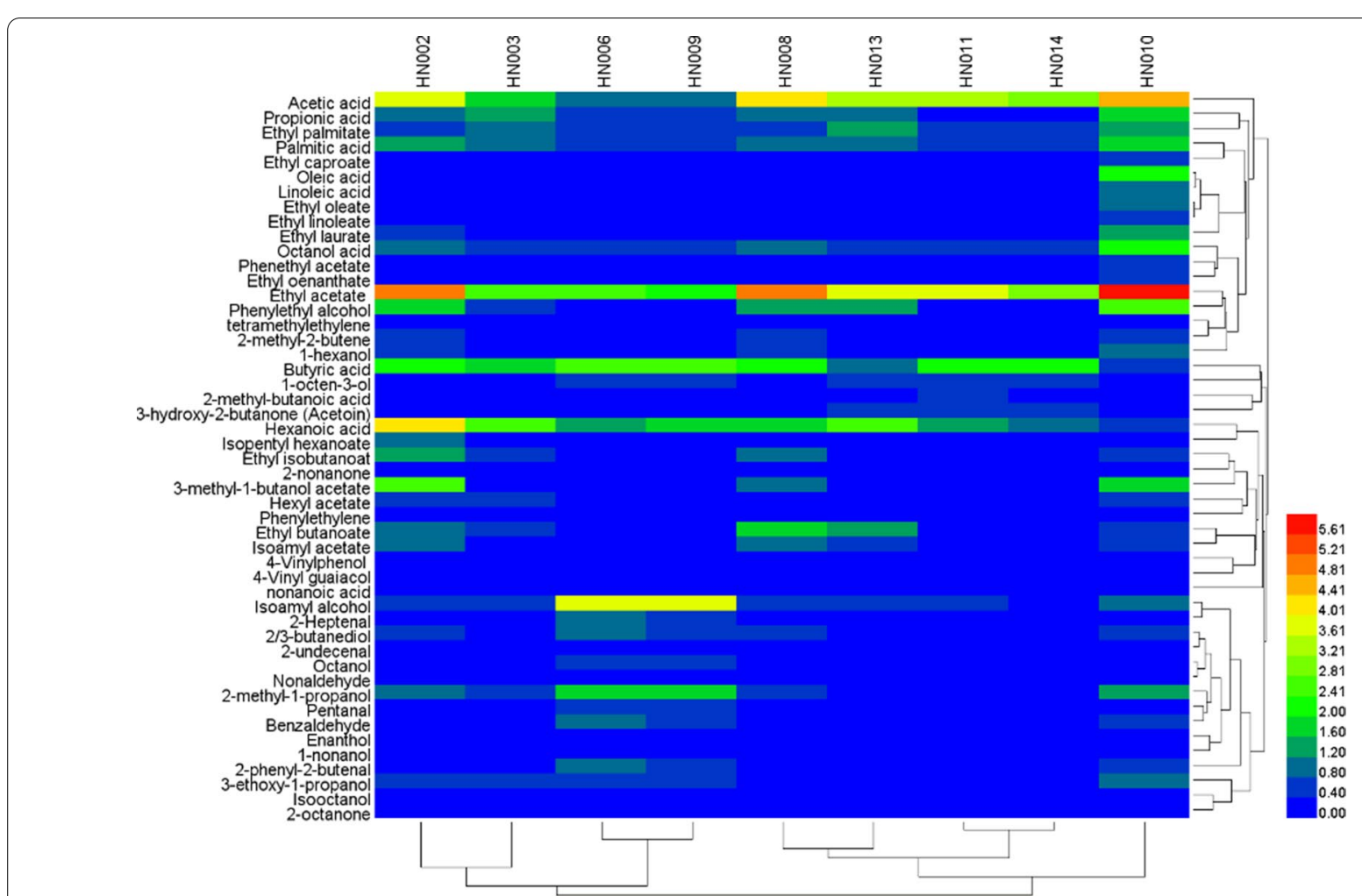

Fig. 2 Heatmap illustrating the differences in the concentrations of volatile compounds in the samples of Chinese chestnut wine prepared with various yeast strains

$0.467 \pm 0.043 \mathrm{~g} / \mathrm{g}$. Thus, we observed that the strain HN006 showed the high fermentation performance of Chinese chestnut wine.

\section{Sensory evaluation}

A sensory evaluation was performed to identify the yeast strain that had the potential to produce a pleasant tasting wine. Sensory characteristics are vital markers to evaluate the wine quality, amongst which sensory appraisal is a commonly accepted method. A significant difference was observed in different quality attributes, such as aroma, after taste, wine taste, mouthfeel, and overall quality (Additional file 1: Figure S3). Among these wine samples, the wine prepared using strain HNO10 was superior to those with other strains based on its highest scores for taste (7.37), aroma (7.76), mouth feel (7.64), after taste (6.90), and overall acceptability (7.12), which agreed with the result of aromatic profile of the volatile compounds determined by SPME-GC-MS. The Saccharomyces strains (HN006, HN009) received the lowest scores in the sensory analysis. Thus, this test identified and verified that the taste-profile of the Chinese chestnut wine made using the yeast strain HN010 was appreciated by the testers and had an affirmative effect on the overall sensory features.
Thus, the non-Saccharomyces strain, HN010, showed the highest amounts of volatile compounds and best sensory characteristics but with poor ethanol fermentation capacity, and the Saccharomyces strain, HN006, showed the highest efficiency in ethanol production and consumption of reducing sugar. Therefore, both these yeast strains were selected to be used as mixed inoculum for producing an improved version of the Chinese chestnut wine. Both strains were deposited in China Center of Industrial Culture Collection (CICC) and assigned the number of CICC1035 (HN006) and CICC1783 (HN010), respectively.

\section{Optimization inoculation sizes for chestnut whiskey brewing}

We tested different inoculation volumes of strains HNO06 and the strain HN010 to inoculate the fermentation mash at the beginning of the fermentation process of the Chinese chestnut wine, and their inoculums were pre-cultured separately as specified in Sect. 2.2. Table 4 lists the effects of the mixed inoculation volumes with different ratios of strains HN006 and HNO10 on the concentration of ethanol and overall sensory scores. We observed an increase in the concentration of ethanol with an increase in the overall combined inoculation volume and 

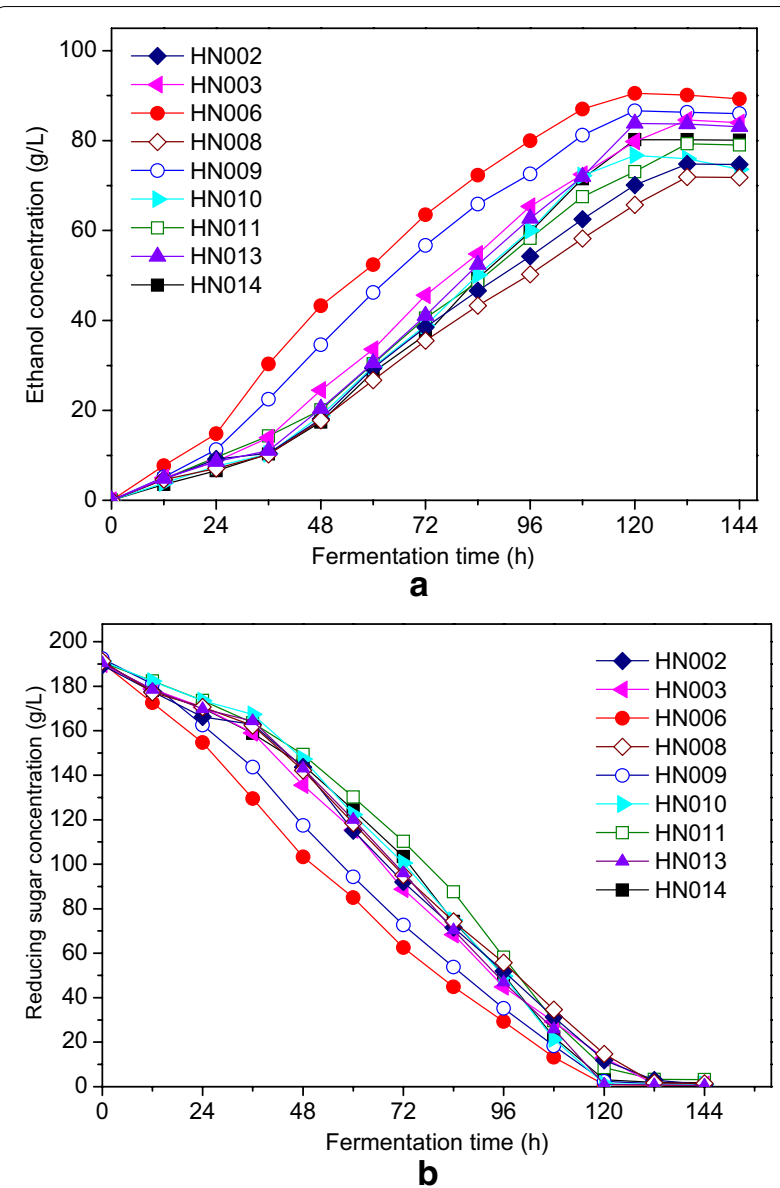

Fig. 3 Fermentation profiles of Chinese chestnut wine with various yeast strains: $\mathbf{a}$ ethanol; $\mathbf{b}$ reducing sugar

reached a maximum value at $6 \%(\mathrm{v} / \mathrm{v})$. Further increase in the inoculation volume resulted in a decrease in ethanol concentration. There was no significant difference in ethanol concentration for different inoculation ratios of HN006 and HN010 at an inoculation size of 5\%. Further modification in the size and ratio of this inoculation resulted in a decreased ethanol production. As for the overall sensory scores, it showed an increasing trend with an increase in the inoculation ratio of the strains HN010 to strain HN006 at various inoculation volumes below 6\%; however, there was no significant difference in the overall sensory scores for various inoculation ratios as the inculcation size reached up to $12 \%(\mathrm{v} / \mathrm{v})$. The highest overall sensory score was also achieved at the mixed inoculation size of $6 \%(\mathrm{v} / \mathrm{v})$ and a HNO06 to HN010 inoculation ratio of $1: 2(\mathrm{v} / \mathrm{v})$. Thus, we used this inoculum size in further studies.

\section{Optimization the ratios of different raw material for chestnut whiskey brewing}

Table 5 illustrates the influence of raw materials with different ratios on the brewing of the Chinese chestnut whiskey. The ratio of chestnut whiskey to malt, and to glutinous rice in the medium had a crucial effect on the sensory score and the production of ethanol.

The data presented in Table 5 revealed an increase in the initial reducing sugar concentration of the saccharified mash with the total amounts of malt and glutinous rice. The ethanol concentration and overall sensory scores increased as the proportion of malt and glutinous rice to Chinese chestnut increased, and both reached a maximum value at the ratio of 5:3:2 (Chinese chestnut: malt: glutinous rice). All multiple-grain fermentations were superior to single-grain fermentation of Chinese chestnut, as shown by a higher overall sensory score and the ethanol content. Table 5 demonstrated that the optimum ratio of chestnut whiskey to malt to glutinous rice, and to corn was 5:2:2:1 (w: w: w: w), which is justified by the highest corresponding value of the sensory score $(8.210 \pm 0.300)$ and highest ethanol concentration $(86.021 \pm 2.470)$. This value will be used for further testing.

Table 3 Evaluation of kinetic parameters for Chinese chestnut wine by various yeast strains

\begin{tabular}{llllcc}
\hline Strain code & $\begin{array}{l}\text { Initial reducing sugar } \\
\text { concentration (g/L) }\end{array}$ & $\begin{array}{l}\text { Fermentation } \\
\text { time (h) }\end{array}$ & $\begin{array}{l}\text { Maximum ethanol } \\
\text { concentration (g/L) }\end{array}$ & $\begin{array}{l}\text { Sugar utilization (\%) } \\
\text { Ethanol yield (g/g) }\end{array}$ & $\begin{array}{l}\text { Volumetric ethanol } \\
\text { productivity (g/L/h) }\end{array}$ \\
\hline HN002 & $189.5 \pm 2.2$ & 132 & $74.9 \pm 2.0$ & $96.0 \pm 3.9$ & $0.40 \pm 0.02$ \\
HN003 & $189.9 \pm 2.0$ & 132 & $84.4 \pm 1.2$ & $99.3 \pm 3.19$ & $0.45 \pm 0.03$ \\
HN006 & $191.6 \pm 2.0$ & 120 & $90.2 \pm 0.9$ & $99.500 \pm 5.6$ & $0.47 \pm 0.04$ \\
HN008 & $191.3 \pm 2.0$ & 132 & $71.9 \pm 1.9$ & $97.5 \pm 2.6$ & $0.38 \pm 0.02$ \\
HN009 & $192.4 \pm 3.4$ & 120 & $86.6 \pm 1.0$ & $98.8 \pm 4.8$ & $0.75 \pm 0.03$ \\
HN010 & $191.2 \pm 3.7$ & 120 & $76.7 \pm 1.1$ & $98.5 \pm 4.6$ & $0.55 \pm 0.03$ \\
HN011 & $190.7 \pm 3.1$ & 132 & $79.3 \pm 1.0$ & $95.5 \pm 3.5$ & $0.40 \pm 0.03$ \\
HN013 & $190.4 \pm 2.5$ & 120 & $83.8 \pm 1.4$ & $98.9 \pm 4.7$ & $0.42 \pm 0.03$ \\
HN014 & $190.6 \pm 2.2$ & 120 & $80.2 \pm 1.8$ & $98.4 \pm 2.4$ & $0.44 \pm 0.03$ \\
\hline
\end{tabular}


Table 4 Effect of different inoculum sizes and ratios of strain HN006 and strain HNO10 on production of Chinese chestnut wine after 6 days of co-culture fermentation

\begin{tabular}{|c|c|c|c|c|c|}
\hline Inoculum size (\%) & $\begin{array}{l}\text { HN006: HN010 } \\
\text { (v/v) }\end{array}$ & $\begin{array}{l}\text { Initial reducing sugar } \\
\text { concentration }(\mathrm{g} / \mathrm{L})\end{array}$ & $\begin{array}{l}\text { Ethanol concentration } \\
\text { (g/L) }\end{array}$ & Ethanol yield $(g / g)$ & $\begin{array}{l}\text { Overall } \\
\text { sensory } \\
\text { scores }\end{array}$ \\
\hline \multirow[t]{3}{*}{3} & $2: 1$ & $157.4 \pm 3.7$ & $65.8 \pm 3.7$ & $0.44 \pm 0.01$ & $7.2 \pm 0.2$ \\
\hline & $1: 1$ & $156.9 \pm 2.9$ & $62.8 \pm 2.9$ & $0.42 \pm 0.02$ & $7.3 \pm 0.2$ \\
\hline & $1: 2$ & $157.2 \pm 2.6$ & $68.6 \pm 2.6$ & $0.40 \pm 0.02$ & $7.4 \pm 0.2$ \\
\hline \multirow[t]{3}{*}{6} & $2: 1$ & $152.7 \pm 2.3$ & $71.0 \pm 3.2$ & $0.47 \pm 0.03$ & $7.3 \pm 0.2$ \\
\hline & $1: 1$ & $152.7 \pm 3.5$ & $70.9 \pm 3.5$ & $0.46 \pm 0.03$ & $7.4 \pm 0.2$ \\
\hline & $1: 2$ & $152.5 \pm 2.2$ & $71.2 \pm 2.2$ & $0.47 \pm 0.02$ & $7.5 \pm 0.2$ \\
\hline \multirow[t]{3}{*}{9} & $2: 1$ & $147.3 \pm 2.6$ & $60.6 \pm 2.6$ & $0.41 \pm 0.02$ & $7.4 \pm 0.3$ \\
\hline & $1: 1$ & $145.7 \pm 3.3$ & $54.7 \pm 3.3$ & $0.39 \pm 0.02$ & $7.5 \pm 0.3$ \\
\hline & $1: 2$ & $146.9 \pm 2.9$ & $55.9 \pm 2.9$ & $0.38 \pm 0.03$ & $7.5 \pm 0.2$ \\
\hline \multirow[t]{3}{*}{12} & $2: 1$ & $142.8 \pm 3.9$ & $52.2 \pm 3.9$ & $0.37 \pm 0.02$ & $7.4 \pm 0.2$ \\
\hline & $1: 1$ & $143.3 \pm 2.6$ & $51.3 \pm 2.6$ & $0.36 \pm 0.02$ & $7.4 \pm 0.2$ \\
\hline & $1: 2$ & $142.3 \pm 2.9$ & $49.6 \pm 2.9$ & $0.35 \pm 0.02$ & $7.4 \pm 0.2$ \\
\hline
\end{tabular}

\pm indicates standard derivation among replicates

Table 5 Effect of different ratios of raw material on production of Chinese chestnut wine after 6 days of co-culture fermentation

\begin{tabular}{llll}
\hline $\begin{array}{l}\text { Raw material ratio (Chinese chestnut: malt: } \\
\text { glutinous rice, w: w: } \mathbf{w})\end{array}$ & $\begin{array}{l}\text { Initial reducing sugar concentration } \\
\text { (g/L) }\end{array}$ & Ethanol concentration (g/L) & $\begin{array}{l}\text { Overall } \\
\text { sensory } \\
\text { scores }\end{array}$ \\
\hline $4: 4: 2$ & $208.6 \pm 3.5$ & $85.5 \pm 2.9$ & $7.8 \pm 0.5$ \\
$4: 3: 3$ & $203.5 \pm 2.9$ & $85.0 \pm 3.5$ & $7.6 \pm 0.4$ \\
$4: 2: 4$ & $198.5 \pm 3.8$ & $86.0 \pm 3.8$ & $7.5 \pm 0.2$ \\
$5: 3: 2$ & $188.7 \pm 2.2$ & $86.0 \pm 2.5$ & $8.2 \pm 0.3$ \\
$5: 2.5: 2.5$ & $181.7 \pm 2.2$ & $84.3 \pm 3.0$ & $7.9 \pm 0.5$ \\
$5: 2: 3$ & $178.2 \pm 2.9$ & $82.5 \pm 2.9$ & $7.8 \pm 0.4$ \\
$6: 3: 1$ & $171.6 \pm 3.5$ & $80.6 \pm 3.5$ & $7.9 \pm 0.5$ \\
$6: 2: 2$ & $164.5 \pm 2.9$ & $77.5 \pm 2.9$ & $7.8 \pm 0.4$ \\
$6: 1: 3$ & $161.6 \pm 3.8$ & $75.6 \pm 3.8$ & $7.7 \pm 0.3$ \\
\hline
\end{tabular}

Table 6 Effect of different supplementation amounts of nitrogen nutrient on production of Chinese chestnut wine after 6 days of co-culture fermentation

\begin{tabular}{lccc}
\hline $\begin{array}{l}\text { Supplementation amounts of yeast extract } \\
(\mathbf{g} / \mathbf{L})\end{array}$ & Initial reducing sugar concentration $\mathbf{( g / L )}$ & Ethanol concentration (g/L) & $\begin{array}{l}\text { Overall } \\
\text { sensory } \\
\text { scores }\end{array}$ \\
\hline 0 & $189.5 \pm 4.0$ & $85.7 \pm 2.2$ & $8.1 \pm 0.4$ \\
2 & $188.6 \pm 4.2$ & $88.6 \pm 3.5$ & $8.3 \pm 0.5$ \\
4 & $190.9 \pm 3.6$ & $90.7 \pm 3.9$ & $8.6 \pm 0.4$ \\
6 & $189.6 \pm 4.1$ & $92.6 \pm 3.9$ & $8.9 \pm 0.3$ \\
8 & $191.6 \pm 3.9$ & $86.8 \pm 2.2$ & $8.8 \pm 0.5$ \\
10 & $190.9 \pm 4.2$ & $78.2 \pm 2.8$ & $8.7 \pm 0.6$ \\
\hline
\end{tabular}




\section{Optimization nitrogen nutrient additions for chestnut whiskey brewing}

Table 6 demonstrates the effect of the addition of various amounts of nitrogen nutrients on the fermentation of the Chinese chestnut wine. We observed an increase in the production of ethanol with an increase in the supplementation amounts of yeast extract. We obtained the maximum concentration of ethanol $(92.587 \pm 3.872 \mathrm{~g} / \mathrm{L})$ was obtained at the yeast extract concentration of $6 \mathrm{~g} / \mathrm{L}$, compared with the fermentation without the addition. As for the overall sensory scores of the obtained wine, we observed a gradual increase with an increase in the amounts of yeast extract added and reached the maximum of $8.910 \pm 0.300$ when $6 \mathrm{~g} / \mathrm{L}$ yeast extract was supplemented in the fermentation system; however, there was no significant change with a further increase in the supplementation of the yeast extract. This phenomenon was attributed to the fact that the appropriate amount of yeast extract promoted aroma production, while an excess induced the growth of the strain but was disadvantageous to the formation of the end product. Thus, both the productivity and the sensory characteristics were improved at $6 \mathrm{~g} / \mathrm{L}$ of yeast extract (Table 6). Further investigation is required to study the impact of the supplementation of nitrogen on the metabolic pathways involved in producing and regulating aroma, as well as to understand how nitrogen affects the process of yeast metabolism in alcoholic fermentation, which will promote an improved utilization of this nutrient to produce volatile compounds.

\section{Pilot-scale fermentation}

After the completion of the fermentation process at the laboratory scale, the optimized parameters were tested in a scaled-up system of $1000 \mathrm{~L}$ reactor to investigate the possibility of industrializing the production of the Chinese chestnut wine. The system was used for producing three batches of Chinese chestnut fermented wine, with an agitation speed of $100 \mathrm{rpm}$ and at a temperature of $30{ }^{\circ} \mathrm{C}$.

The Fig. 4 demonstrates the typical profiles of total cell growth, reducing sugar consumption and ethanol formation over time in the $1000 \mathrm{~L}$ bioreactor. After the initial 12-h lag phase, we observed an increase in the process of both reducing sugar consumption and ethanol production. The maximal growth for strain HN006 $\left(2.065 \times 10^{8} \mathrm{CFU} / \mathrm{mL}\right)$ and the strain HN010 $\left(4.201 \times 10^{8} \mathrm{CFU} / \mathrm{mL}\right)$ was observed after $48 \mathrm{~h}$ and $36 \mathrm{~h}$ of fermentation, respectively. The highest concentration of ethanol was $190.56 \mathrm{~g} / \mathrm{L}$, with a corresponding yield of $0.487 \mathrm{~g} / \mathrm{g}$. Over a $3-\mathrm{d}$ period, we successfully completed three runs using the co-culture fermentation. In

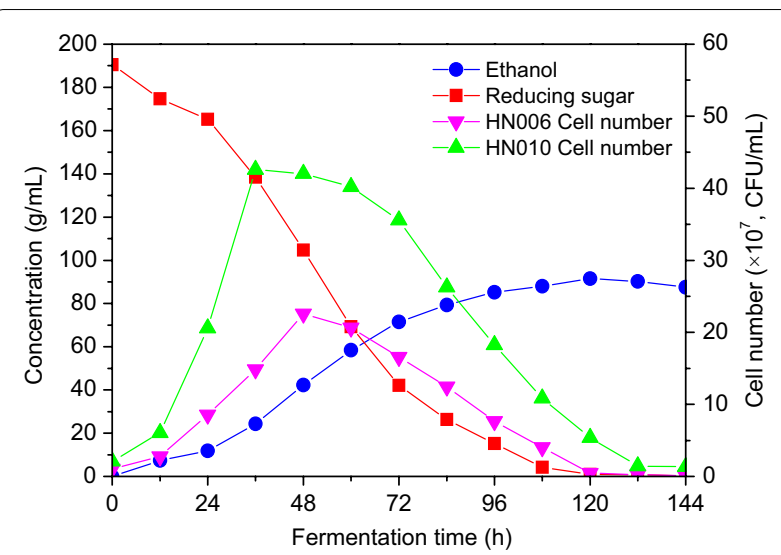

Fig. 4 1000L fermentation profiles of Chinese chestnut wine with co-culture of strain HNOO6 and strain HN010

these three experiments, we observed the key fermentation parameters: final ethanol concentration, volumetric ethanol productivity, and ethanol yield to be consistent. The fermentation performance was successfully demonstrated in the $1000 \mathrm{~L}$ pilot-scale bioreactor and would be suitable for the industrial co-culture fermentation Chinese chestnut wine.

\section{Discussion}

The flavour of distilled wine depends not only on the raw materials and manufacturing technique, but also on the characteristics of yeast and other microorganisms (Alicia et al. 2018). Yeasts are important and can be detected during the whole manufacturing process. Not only do they directly determine the fermentation rate, but they can also transform nutrients into a wide variety of volatile flavour compounds (Ayşe et al. 2018; Wang et al. 2019). Therefore, yeasts are one of the key factors affecting the flavour type and product quality of whiskey (Shi et al. 2019; Wu et al. 2012).

At present, most whiskeys are fermented by Saccharomyces cerevisiae. Although the single fermentation mode by Saccharomyces cerevisiae obtains high alcohol content, but it has the phenomenon of homogenization of whiskey flavor. Recently years, a large number of studies have shown that non-Saccharomyces cerevisiae can produce alcohols, esters, acids, alkanes, aromatic hydrocarbons, ketones and other metabolites in the fermentation process, which can enhance the flavour and enrich the taste of the wine, however, it normally showed poor ethanol fermentation capacity. Nowadays, co-fermentation of selected non-Saccharomyces yeasts with $S$. cerevisiae received more and more acceptance to avoid the defects of pure fermentation and spontaneous fermentation (Luan et al. 2018). Remarkably, the application of 
mixed cultures of Saccharomyces cerevisiae and non-Saccharomyces cerevisiae has been reported to make important contributions to the formation of aroma substances, and also with high efficiency of ethanol production (Yan et al. 2019a, b). Additionally, the use of different species of yeast has proved to improve the percieved flavour in wine (Alicia et al. 2018). Therefore, the selection of yeast strains is critical for the formation of wine aroma, which is one of the key attributes affecting consumer acceptance (Wang et al. 2019). Yuan et al. (2019) selected one excellent non-Saccharomyces cerevisiae strain from 6 aroma-producing yeast strains and co-cultured with $\mathrm{Sac}$ charomyces cerevisiae for Yali fruit wine fermentation and the results demonstrated that the mixed fermentation helped to enhance the aroma and improve the complexity and characteristics of the wine.

In present study, a novel whiskey using Chinese chestnut as the main raw material was developed. First, we isolated the yeast strains and investigated their potential to produce effective Chinese chestnut wine, and the results demonstrated that the strain HN010, which showed the maximum production of the volatile compounds and the best sensory characteristics, along with the strain HNO06 that possessed the highest efficiency in ethanol production were adopted as the most promising yeasts as they combined satisfactory Chinese chestnut wine fermentation with an interesting flavor profile. Further optimization of the Chinese chestnut wine fermentation using a co-culture of strain HNOO6 and strain HN010 demonstrated that the optimum conditions for chestnut wine production were achieved at a mixed inoculum volume of $6 \%(\mathrm{v} / \mathrm{v})$ with an HN006/HN010 inoculation ratio of 1:2 (v/v), a raw materials ratio of 5:3:2 (chestnut whiskey: malt: glutinous rice), and a yeast extract concentration of $6 \mathrm{~g} / \mathrm{L}$. Additionally, a scaled-up fermentation of the Chinese chestnut wine was successfully carried out in a $1000 \mathrm{~L}$ pilot-scale system. This study is the first report to explore the fermentation of Chinese chestnut wine using a mixed culture system. The co-culture of strain HN006 and strain HNO10 showed potential application in the Chinese chestnut wine fermentation process. Additionally, the methods used in this study could provide a generalized experimental scheme to initiate large-scale studies for the identification of useful microbes for the fermentation of wine as well as other food products.

\section{Supplementary Information}

The online version contains supplementary material available at https://doi. org/10.1186/s13568-020-01175-4.

Additional file 1: Figure S1. The manufacturing process of Chinese chestnut whiskey raw wine. Figure $\mathbf{S 2}$. The layout of the $1000 \mathrm{~L}$ Chinese chestnuts wine fermentation pilot plant. 1, Soaking rice cans; 2, Steamed rice cooker; 3, Liquefied tank; 4, Pump; 5, Malt miller; 6, Saccharifying tank; 7, Pump; 8,Filter tank; 9, Pump; 10, Plate heat exchanger; $11,10 \mathrm{~L}$ seed preparation reactor; 12 , Pump; 13, 100L seed preparation reactor; 14, Pump; 15, 1000 L reactor; 16, Pump. Figure S3. Sensory evaluation of Chinese chestnut wine samples by different yeast strains.

\section{Acknowledgements}

This study was financially supported by the Key Research and Development Project of Anhui Province (Grants 1804a07020122, 1704f0704067), the scientific research project of Anhui Provincial Higher Education Institutes (KJ2020a0375), the scientific research project of Anhui Polytechnic University (xjky2020064), and the academic funding for top talents in disciplines (Specialties) of Anhui Provincial Higher Education Institutes (Grant gxbjZD35).

\section{Authors' contributions}

LWZ, SCE, GJQ, GF, and YSB designed the experimental program, participated in the examination and drafted the manuscript. YSB (Corresponding author) is responsible for this study, participated in its design and help to draft the manuscript. All authors read and approved the final manuscript.

\section{Funding}

Not applicable.

\section{Availability of data and materials}

Please contact author for data requests.

\section{Ethics approval and consent to participate}

Ten trained investigators gave informed consent and agreed to participate in the study for sensory evaluation.

\section{Consent for publication}

Not applicable.

\section{Competing interests}

The authors declare that they have no competing interests.

\section{Author details}

${ }^{1}$ College of Biological and Food Engineering, Anhui Polytechnic University, Anhui 241000, People's Republic of China. ${ }^{2}$ School of Life Science, Huainan Normal University, Huainan, Anhui 232001, People's Republic of China. ${ }^{3}$ Anhui Yingjia Group Co., Ltd, Luan, Anhui Province 237271, People's Republic of China.

Received: 9 October 2020 Accepted: 28 December 2020

Published online: 11 January 2021

\section{References}

Alicia G, Teun B, Zoran G, Michael K (2018) Evaluation of non-Saccharomyces yeasts in the fermentation of wine, beer and cider for the development of new beverages. J Inst Brew 124:389-402

Annalisa R, Martina C, Alessia L, Chiara DA, Gianni G, Camilla L (2018) Volatile profile of elderberry juice: effect of lactic acid fermentation using $L$. plantarum, L. rhamnosus and L. casei strains. Food Res Int 105:412-422

Aslankoohi E, Herrera-Malaver B, Rezaei MN, Steensels J, Courtin CM, Verstrepen KJ (2016) Non-conventional yeast strains increase the aroma complexity of bread. PLOS ONE 11(10):2-18

Ayşe Y, Emine M, Enes D (2018) Microbial species playing roles for the production of traditional Kasar cheese during pre-maturation period. LWT-Food Sci Technol 91:406-413

Du H, Wang XS, Zhang YH, Xu Y (2019) Exploring the impacts of raw materials and environments on the microbiota in Chinese Daqu starter. Int J Food Microbiol 297:32-40

Jacques N, Mallet S, Laaghouiti F, Tinsley CR, Casaregola S (2017) Specific populations of the yeast Geotrichum candidum revealed by molecular typing. Yeast 34:165-178

Laura C, Mark S, Francesca C, Maurizio C, Cristian V (2019) Volatile profile of reduced alcohol wines fermented with selected non-Saccharomyces yeasts under different aeration conditions. Food Microbiol 84:103247 
Luan Y, Zhang BQ, Duan CQ, Yan GL (2018) Effects of different pre-fermentation cold maceration time on aroma compounds of Saccharomyces cerevisiae co-fermentation with Hanseniaspora opuntiae or Pichia kudriavzevii. LWT-Food Sci Technol 92:177-186

Marycarmen UV, Jacobo RC, Carlos HAA, Anne G, Eugenia LC (2020) Analysis of volatile compounds of five varieties of Maya cocoa during fermentation and drying processes by Venn diagram and PCA. Food Res Int 129:108834

Miller GL (1959) Use of dinitrosalicylic acid reagent for determination of reducing sugar. Anal Chem 1:426-428

Sheng JW, Yu L (2015) Preparation and characterisation of the oligosaccharides derived from Chinese water chestnut polysaccharides. Food Chem 185(15):15-18

Shi WK, Wang J, Chen FS, Zhang XY (2019) Effect of Issatchenkia terricola and Pichia kudriavzevii on wine flavor and quality through simultaneous and sequential co-fermentation with Saccharomyces cerevisiae. LWT-Food Sci Technol 116:108477

Simonetta V, Valentina A, Antonio V, Paola D, Luca C (2016) Molecular identification and physiological characterization of yeasts, lactic acid bacteria and acetic acid bacteria isolated from heap and box cocoa bean fermentations in West Africa. Int J Food Microbiol 216:69-78

Wang DQ, Chen LQ, Yang F, Wang HY, Wang L (2019) Yeasts and their importance to the flavour of traditional Chinese liquor: a review. J Inst Brew 125:214-221

Wu Q, Xu Y, Chen L (2012) Diversity of yeast species during fermentative process contributing to Chinese Maotai-flavour liquor making. Lett Appl Microbiol 55(4):301-307

Yan S, Dong D (2019) Main parameters and the dynamics of volatile compounds during the fermentation of Chinese Mao-tofu from Huangshan region. Food Sci Biotechnol 28:1315-1325
Yan S, Chen X, Wu J, Wang P (2013) Pilot-scale production of fuel ethanol from concentrated food waste hydrolysates using Saccharomyces. Bioproc Biosyst Eng 36:937-946

Yan S, Chen X, Xiang X (2019a) Improvement of the aroma of lily rice wine by using aroma-producing yeast strain Wickerhamomyces anomalus HN006. AMB Express 9:89

Yan SB, Tong QQ, Guang JQ (2019b) Yeast dynamics and changes in volatile compounds during the fermentation of the traditional Chinese strongflavor Daqu. LWT-Food Sci Technol 106:57-63

Yuan XL, Bian MH, Liu MM, Zou YF, Xu Q (2020) Optimization of fermentation conditions of Yali wine with Saccharomyces cerevisiae co-inoculated with aroma-producing yeast. Food Ferment Ind 46(6):184-190 (In Chinese)

Zhang L, Gao HY, Baba M, Okada Y, Okuyama T, Wu LJ, Zhan LB (2014) Extracts and compounds with anti-diabetic complications and anti-cancer activity from Castanea mollissina Blume (Chinese chestnut). BMC Complem Altern M 14:422

Zhang BQ, Luan Y, Duan CQ, Yan GL (2018a) Use of Torulaspora delbrueckii Co-fermentation with two Saccharomyces cerevisiae strains with different aromatic characteristic to improve the diversity of red wine aroma profile. Front Microbiol 9:606

Zhang L, Wang ZG, Shi GY, Yang H, Wang XM, Zhao HY, Zhao SH (2018b) Effects of drying methods on the nutritional aspects, flavor, and processing properties of Chinese chestnuts. J Food Sci Tech Mys 55(9):3391-3398

\section{Publisher's Note}

Springer Nature remains neutral with regard to jurisdictional claims in published maps and institutional affiliations.

\section{Submit your manuscript to a SpringerOpen ${ }^{\odot}$ journal and benefit from:}

- Convenient online submission

- Rigorous peer review

- Open access: articles freely available online

- High visibility within the field

- Retaining the copyright to your article

Submit your next manuscript at springeropen.com 